\title{
A review on content-based image retrieval representation and description for fish
}

\begin{abstract}
There is an increasing interest in the description and representation of fish species images. For that purpose, Content-based Image Retrieval (CBIR) is applied. Due to the uncontrolled deep sea underwater environment, it is very hard to accurately estimate the similarities between the fishes and retrieves them according to its species due to ineffective visual features extraction for fish image representation. In this paper, CBIR for representation and description of fish is reviewed. Shape is one of the most important features to describe fish. This paper considers the combination of global and local shape features. Existing combination is carefully studied and the importances of global and local shape features are presented. The focus of possible future works is also suggested.
\end{abstract}

Keyword: Content-based image retrieval; Feature extraction; Fish; Global feature; Local feature; Shape 